\title{
A methodology for developing product platforms in the specific setting of the housebuilding industry
}

\author{
Vanessa S. Veenstra · Johannes I. M. Halman • \\ Johannes T. Voordijk
}

Received: 8 October 2005/Revised: 21 March 2006/ Accepted: 6 September 2006/

Published online: 3 November 2006

(C) Springer-Verlag London Limited 2006

\begin{abstract}
Platform based strategies have proved to be a successful approach for achieving optimum balances between standardization and variation in many industries. However, application of this concept in the housebuilding industry is relatively new. This article describes a new methodology for developing product platform architectures in the specific setting of the housebuilding industry. This methodology comprises a reference framework describing the basic elements that constitutes a product platform, supported by a protocol for developing product platforms. The applicability of the proposed methodology has been tested at a Dutch housebuilding company. In this study, the methodology demonstrated its added value in determining which modules to standardize and defining a product platform. This article also describes a distinctive method of housing classification that is based on the spatial use of houses. Compared to the traditional classification system based on technical construction elements, the proposed new classification system facilitates a better translation of functional requirements into technical specifications.
\end{abstract}

Keywords Product platform - Product architecture . Standardization · Modularization · Customization . Housebuilding industry

V. S. Veenstra · J. I. M. Halman $(\bowtie)$.

J. T. Voordijk

Faculty of Engineering Technology,

Department of Construction Management and Engineering,

University of Twente, PO Box 217, 7500 AE Enschede,

The Netherlands

e-mail: j.i.m.halman@utwente.nl

\section{Introduction}

Nowadays, in many industries, a company's ability to develop single products efficiently and effectively is not enough to stay competitive. Customers are demanding products and services that match their individual preferences and tastes. What used to be a predominant demand for standard mass-market products has fragmented into demands for different "flavours" of similar products. However, producing such products can be expensive and increasing competition forces companies to moderate prices. In this competitive environment, companies are seeking means to balance standardization and variation in their products. Customer-focused design strategies based on the creation of product families sharing common module-based platforms currently receive wide interest. In industries as diverse as electronics, software, automobile and domestic appliances, module-based product platforms have already proved successful (Fixson 2002; Martin and Ishii 2002; Salvador et al. 2002; Muffato and Roveda 2000). The benefits are mainly greater flexibility in product design, efficiency in product development and realization, and effectiveness in both communication and market positioning (Halman et al. 2003). The key elements of a successful product or service-in terms of its features, how quickly it is developed, and the cost to produce it - are based on the provider's ability to create robust, shared platforms that are leveraged into many products (Meyer and DeToreb 2001).

Although studies on product platforms have increased considerably in the last decade, their application to the building industry still remains an underresearched area. However, in the housebuilding 
industry we note developments that also encourage building firms to consider innovative platform-based approaches (e.g. Gibb 2001; Roy et al. 2003). During the 1960s and 1970s, housebuilders primarily focused on the mass production of their products. However, today's consumers are no longer satisfied with undifferentiated products, even though the products themselves are good in terms of product quality (Noguchi 2003). Therefore, the housebuilding industry is currently looking for ways to increase the influence of customers on the design of their own houses, without increasing the price to a level not accepted by target consumers and loosing the advantages of serial, project-wise, production. Housebuilding firms in countries such as Japan, the USA, Germany, the UK and The Netherlands are starting to see the potential of adopting more customer-focused building concepts. Increasing competition, a growing demand for variety and high inhabitant mobility are some of the forces that make housebuilding firms consider new types of product and process design. The degree of industrialisation of the Japanese housebuilding industry has always been high compared with that in Europe (Gann 1996; Barlow et al. 2003). It has also achieved a high degree of customer focus. In both Japan and the USA, conditions are favourable for the customer-driven and modular production of houses because of the significant nationwide harmonisation of regulations and building methods for light structures (Kendall 1999). In Germany, the off-site manufacturing industry offers customer focused housing concepts that are associated by customers with high quality and considerable variety and flexibility in external features (DTI 2004). In the UK, partnerships between housebuilders and customers are receiving increased attention (Ball 1999; Barlow et al. 2003; Craig and Roy 2004; Ozaki 2003). In The Netherlands, the flexible design model has become popular in the house building market. This model incorporates a number of predefined options from which consumers are able to choose (Van den Thillart 2004; Wolters 2001; Voordijk et al. 2006).

In this article, we present a methodology for developing product platform architectures in the specific setting of the housebuilding industry. This methodology is based on the product platform structure theories developed by Ulrich (1995), Baldwin and Clarke (2000), Hofer and Halman (2005) and the design for variety method (DFV) developed by Martin and Ishii (2002).

The structure of the rest of this article is as follows. First, a theoretical background is presented of the basic concepts that are used in the design and application phase of product platforms in industry. In that section we will also explain our research framework. This section is followed by an outline of the research methodology and the successive steps that were followed in the empirical study. An extensive presentation of the study results is given in the next section. Finally, the contributions to literature, managerial implications, limitations and future work are discussed.

\section{Theoretical background and research framework}

Platform thinking is defined as the process of identifying and exploiting commonalities among a firm's offerings, target markets, and the processes for creating and delivering offerings (Halman et al. 2003). A product platform can be defined as a set of subsystems and interfaces that form a common structure from which a stream of derivative products can be developed and produced efficiently (Sawhney 1998). The stream of derivative products comprises the product family. A product family is the collection of products that share the same platform(s). The principle behind the platform concept is to achieve an optimum balance between the commonality potential and the differentiation needs within a product family. A basic requirement therefore is the decoupling of elements to achieve the separation of common (platform) elements from differentiating (nonplatform) elements (Halman et al. 2003).

The platform approach has been widely advocated in literature (e.g. Jones 2003; Krishnan and Gupta 2001; Meyer and Lehnerd 1997; Meyer et al. 1997) as an option to create desirable variety at a cost acceptable to the consumer. The expected benefits include greater flexibility in product design, efficiency in both product development and realization, and effectiveness in both communication and market positioning. However, unlike the benefits, the limitation and costs related to product family development have not yet been addressed widely and specifically in the literature. In most cases, developing the initial platform requires greater investment and more development time than developing a single product, potentially delaying the time to market of the first product and lengthening the payback time. Meyer et al. (1997) introduced two new measures which they termed platform efficiency and platform effectiveness, for measuring the performance of product families. Platform efficiency is the degree to which a platform allows the economical development of derivative products. Mathematically, platform efficiency $=(R \& D$ cost of derivative product $) /(R \& D$ cost of platform version). Platform effectiveness is the degree to which the products based on common product 
platform produce revenue for the firm relative to the cost of developing such products. Mathematically, platform effectiveness $=$ (net sales of derivative products)/(development costs of derivative products). These measures, when combined with visual interpretation of product family maps, can also help management decide on the timing of product platform renewal and the frequency of derivative product developments using existing product platforms (Meyer and Utterback 1992).

Developing product platforms self-evidently requires knowledge and information about their structure. There are several views on the structure of product platforms. Baldwin and Clark (2000) define three aspects: the architecture, the interfaces and the standards. The architecture comprises the specification of the modules that constitute the platform, and their functions. The interfaces constitute the scheme by which the modules interact and communicate, and the standards are the design rules that the modules must conform to. In product development literature, modules have been defined as common, standardized and compatible parts with a specific function (Sanchez and Mahoney 1996; Schilling 2000; Baldwin and Clark 2000; Wolters 2001). Hofer and Halman (2005) describe a similar relation between modules and functions, which they term product architecture. However, they also distinguish four layers to separate differentiation needs and commonality potential within a product family. The four layers are: component variety, functional variety, system layout, and system integration. The component variety layer describes those building blocks of a product which are needed for basic system performance. The second layer addresses the functional description of the system which is realized through the combination of components or building blocks into subsystems. These first two layers define the subsystems, which are arranged in a system layout (third layer) so as to perform in the desired way. The integration of these subsystems in their defined layout covers the fourth layer of product architecture. Ulrich (1995) defines the architecture of a product as: (1) the arrangement of functional elements; (2) the mapping from functional elements to physical components; and (3) the specification of the interfaces among interacting physical components.

Ulrich furthermore makes a distinction between two types of product architecture, namely modular and integral. A modular architecture includes a one-to-one mapping from functional elements in the function structure to the physical components of the product, and specifies de-coupled interfaces between components. An integral product architecture includes a complex (rather than a one-to-one) mapping from functional elements to physical components and/or coupled interfaces between components. Normally, a change made to one component requires a change to the other components for the correct functioning of the total product. In contrast, in modular product architecture, components are interchangeable, autonomous, loosely coupled, individually upgradeable and the interfaces are standardized. Wolters (2001) defines five features that are of importance in determining the degree of modularity: (1) distinctiveness/autonomy of modules; (2) loose coupling between modules; (3) tight coupling within modules; (3) clarity of mapping between functions and modules; (4) standardization of interfaces; and (5) low levels of coordination (selforganization; coordination embedded in the architecture). Such a modular architecture allows a design change to be made to one module without requiring a change to other modules for the product to still function properly.

The predominant approach to platform-based product development, is through the development of a module-based product family in which product family members are instantiated by adding, substituting, and/ or removing one or more functional modules from the platform. An alternative approach is through the development of a scale-based product family in which one or more scaling variables are used to "stretch" or "shrink" the platform in one or more dimensions to satisfy a variety of market niches (Simpson et al. 2006).

According to Voordijk et al. (2006), modularization allows products to be differentiated to a high degree and thus meet varied customer requirements. Besides generating product variety, a range of other module drivers have been described as reasons for the grouping of technical solutions or parts within modules. Erixon (1998) lists twelve reasons to group product elements into modules, namely development, variety, production, procurement, quality and after sales.

To develop a methodology for developing product platform architectures in the specific setting of the housebuilding industry, a reference framework is needed to describe those basic elements that constitute a product platform (see Fig. 1).

\section{Determine the product architecture.}

To determine the product architecture, it is first necessary to specify the modules by defining their respective functions. Next, the physical elements by which each module will fulfil its intended function need to be determined. The third step in product architecture determination is to determine the layout of the modules. 
Fig. 1 Protocol for

developing product platforms

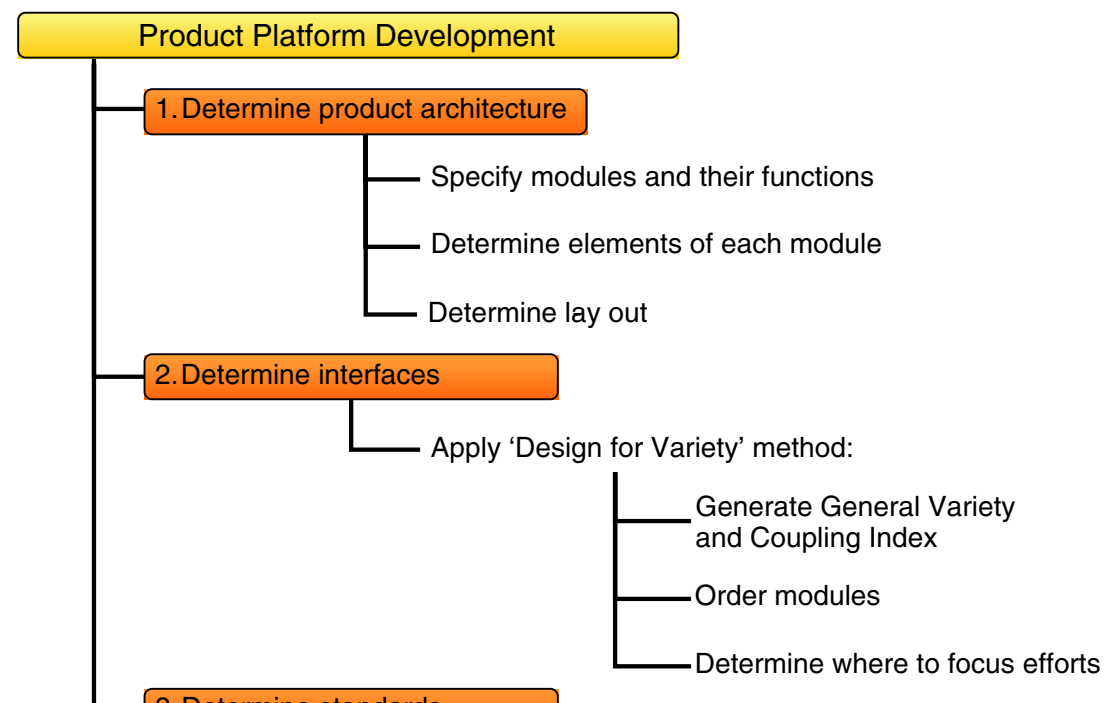

3. Determine standards
To specify the modules and their functions in the specific setting of the housebuilding industry, a clear understanding of the structure and parts of the final building is needed. In this respect, one could classify a building not only according to its technical construction elements (e.g. load-bearing elements, building physics elements) but also according to its various spatial uses (e.g. multifunctional rooms, rooms with a specific use, secluded rooms, supplementary rooms). A housebuilding company would be inclined to classify a building according to its technical construction elements, in contrast to the customers' spatial perspective of a building. To improve communication between the housebuilding industry and its customers and translating the customers' needs, it is suggested that the modules and their functions should be specified according to their spatial use. Subsequently, the construction elements of each module can be determined as well as the relationships between modules leading to specifications of layout needs. In this way, functional requirements can be directly translated into technical specifications.

\section{Determine the interfaces}

A thorough examination of platform interfaces is part of the 'design for variety' method (DFV) developed by Martin and Ishii (2002). This method is a detailed, step-by-step approach to aid design teams in developing a product platform architecture that incorporates standardization and modularization which aims to reduce future design costs and efforts. DFV consists of three main steps. The first DFV step is to generate the general variety index (GVI) and the coupling index (CI). The GVI is a measure for the amount of redesign effort required for future designs of the product. The CI is a measure of the coupling among the product components. The second DFV step is to order the modules based on the results of the previous DFV step. Ordering the modules helps the design team to determine where to concentrate their efforts, where to standardize and where to modularize, which is the third DFV step.

\section{Determine the standards}

In the third and final protocol step, the standards-the design rules that the modules conform to-are determined, based on the information from the preceding steps. In this way, the design team can develop a decoupled architecture that requires less design effort for follow-on products.

\section{Research methodology}

This section describes the successive steps that were followed in this study.

As explained earlier, the objective of this research has been to develop a methodology for developing product platform architectures within the specific setting of the housebuilding industry. A preliminary phase was spent defining the research objectives, conducting a literature review about product platforms and modularization, as well as interviewing experts in the field of house building and analysing current developments in the housebuilding industry in general, and those in The Netherlands specifically. These preliminary studies helped us to understand the theory and basic concepts that are used in the design and application phase of 
product platforms in industry. Based on the specific characteristics of the building industry, we developed the research framework and protocol as outlined in the previous section.

In the following phase of the research, this framework was applied and the applicability of the proposed protocol was tested at Plegt-Vos Living. An important reason for selecting this firm was its past performance in developing and offering customized housing concepts. Plegt-Vos Living is a division of Vos Construction Division, a developing building company operating in the north, east and central parts of The Netherlands. This company employs about 550 people and has annual revenues of about 140 million euros. To address the differentiation in demand, in 2003 PlegtVos Living started the development of a model from which several distinctive types of houses could be derived. Every type starts with minimum standard measures. At the start of this research, the model was still in its conceptual state. Within the company, it became essential to better define the model and frame the components, which gave rise to the start of this indepth research which lasted a year. To collect data, document studies were undertaken, employees of every department at Plegt-Vos Living were interviewed and a total of 31 committee meetings for components management were observed. The committee meetings were attended by the two deputy managing directors, the plan preparation and design staff and project management staff. All were also involved in discussing and testing the applicability of the research framework and protocol.

\section{Plegt-Vos Living: applying the research framework}

Our research framework was used to develop a product platform for the design concept adopted by Plegt-Vos Living. We present the results of this study, following the protocol outlined in the research framework.

\subsection{Determine the product architecture}

\subsubsection{Specify the modules and their functions}

Plegt-Vos Living designed a few types of houses, each with a different hallway. Each hallway included a standardized entrance, stairs, a toilet and a utilities meter box. However, the layout and location of the hallway differ for each type of house. For instance, a few of the house types have a front entrance; others a side entrance. Furthermore, the location of the technical installations such as the central heating system on the top floor is related to the hallway. The technical installations and the hallway comprise the standard core of the house. The main reason that Plegt-Vos Living selected this as the central part of each house is to centralize the piping and electric wiring. This central part can be specified as the traffic module with its function being to connect rooms on all floors and to provide central facilities for channelling electric wiring, data cabling and water piping.

Furthermore, Plegt-Vos Living uses two materials for the main structure: concrete and lime-sand stone. These two materials are interchangeable. To achieve this interchangeability they established standard measures. This structure module consists basically of the foundation construction, floors and external walls and has a load bearing function. The traffic module and the structure module together form the base level of the house.

All other rooms are connected to the traffic module, for example, the kitchen with its function of providing space with food preparation facilities and the bathroom providing space with washing facilities. Plegt-Vos Living uses a systems wall in the bathroom. The pipe works are integrated in this wall and sanitary ware can easily be connected. Actually, the bathroom is part of the central part of the house and its location is fixed. The systems wall is an extra utility to centre the conduit. However, in one of the housing types, the Long Life house, the bathroom is placed on the ground floor. In this housing type, the location of the bathroom is also fixed, but since the location of the bathroom differs among the housing types, the bathroom is considered as a distinct module. Besides the bathroom and kitchen, there are other room modules such as bedrooms and the living room. All these room modules can be referred to as built-in modules. The built-in modules are connected to the traffic module and placed into the structure module. Besides the builtmodules, Plegt-Vos Living also offers several extension modules, for instance a garage and a storage area. The building can furthermore be extended by placing a bay window and/or dormer on ground respectively roof level. Besides the option to widen and lengthen the whole house, Plegt-Vos Living also offers the option to extend the house on the ground level. The function of this module is extending the building on ground level to lengthen the living room. The built-in and extension modules together define the floor plans of the house.

The exterior of the house is like a skin or shell around the modules with the function of embellishing the house on the outside. This exterior module contains parts that determine the appearance of the building, in terms of the roof shape, the sides, windows 
and doors. Plegt-Vos Living tries to offer as much choice to the customer in architecture as possible. By offering a choice of module types and module combinations, the customer can individualize the house. Of course, this also applies to the interior. The interior module embellishes the house on the inside. Elements include floor-, wall- and ceiling finishing and internal doors. The interior and exterior together form the finish. An overview of the Plegt-Vos Living modules is shown in Fig. 2.

\subsubsection{Determine the elements of each module}

After identification of the modules and their functions, further specification is achieved by focusing on the elements of the modules. The NL-SfB code is used to classify the elements, since this code is intended for construction elements. The NL-SfB code is not only applicable to housing construction, but also to building construction in general. NL-SfB is the Dutch version of the international SfB classification system. SfB classifies building parts for cost calculation and work specification purposes. The system has been in use for more than 30 years.

In this research, only the elements applicable to housebuilding are used. Elements such as building maintenance facilities and transport facilities (such as elevators and escalators) are not used.

Table 1 shows an overview of the elements in each module. For instance, the room module contains the
Fig. 2 Specification and hierarchy of the modules
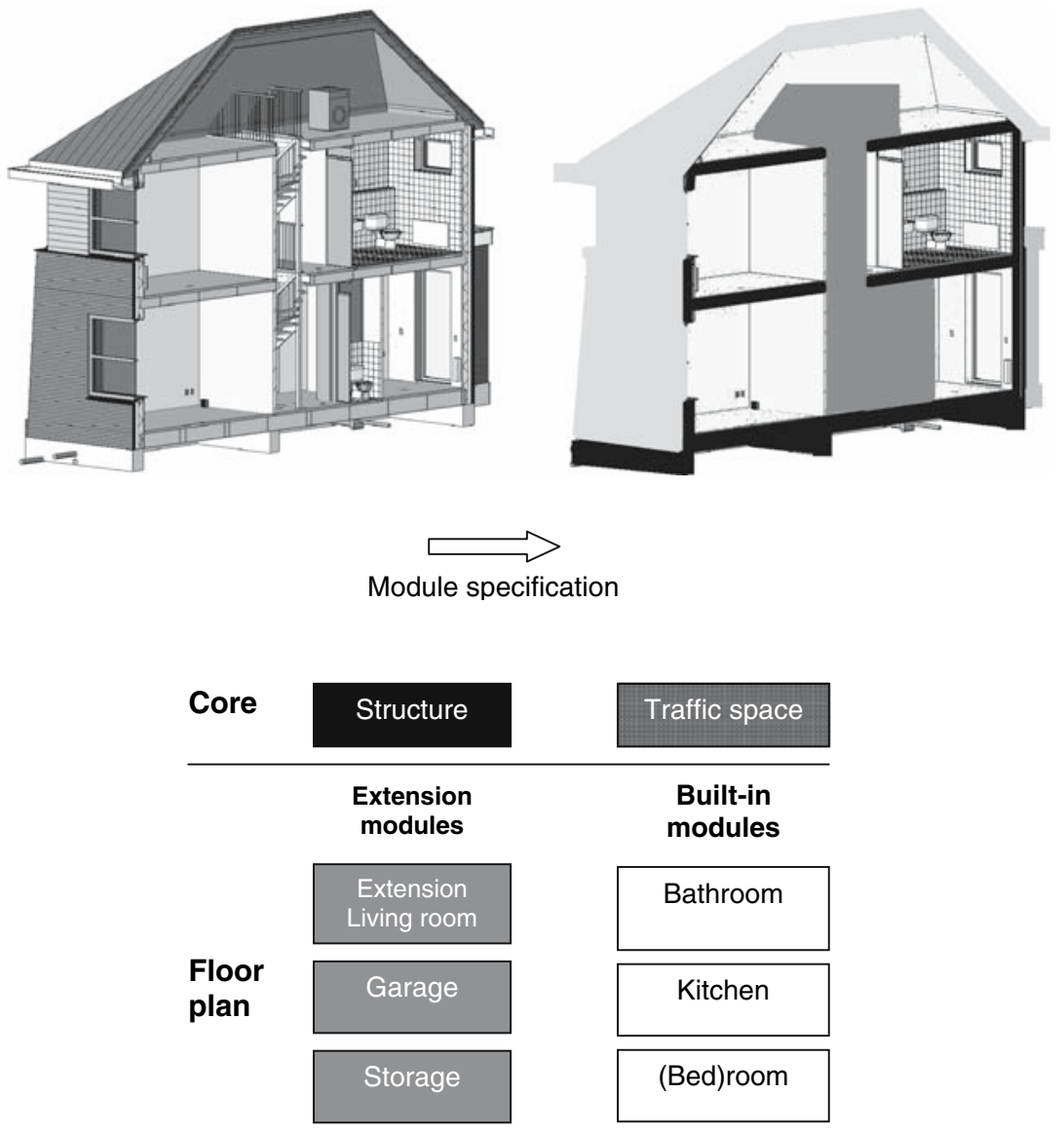

Bay window

Dormer

Finishes

Exterior (building style) 
Table 1 Housing modules and their elements

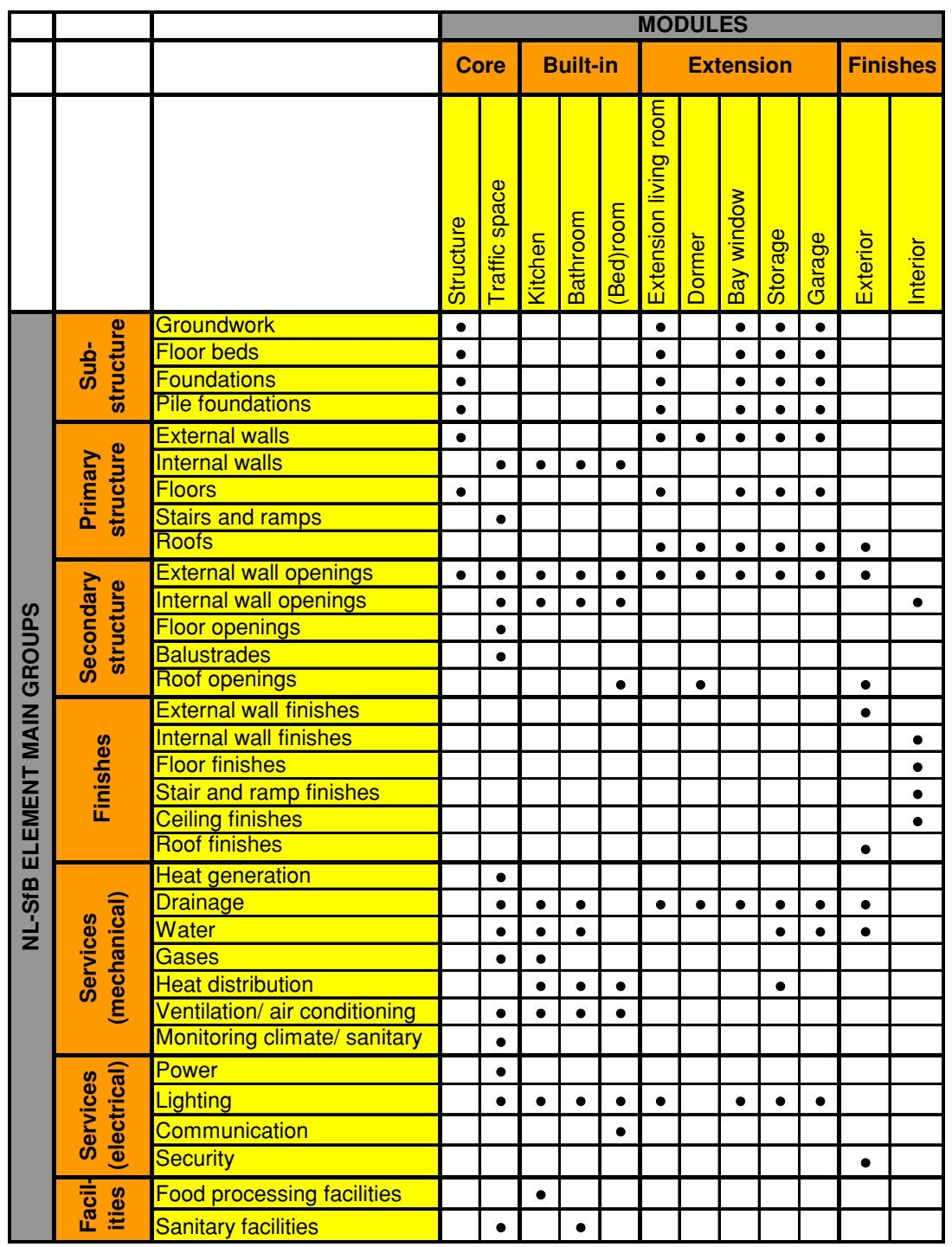

elements: internal walls, external wall openings (windows), internal wall openings (room door), roof openings (windows), heat distribution (radiator), ventilation, lighting (switches, electric sockets, etc.) and communication (e.g. internet). No distinction is made between living rooms, bedrooms, study room, etc. since all modules consist of the same type of elements. The purpose of the room is defined by the resident, who furnishes the room to match the purpose of the room. The table is to be read by column. For instance, external walls in the structure module are the external walls of the building. These external walls are different from the external walls of the garage. However, the information in the rows give an idea of the elements that are applied in several modules. For instance, the external wall openings and the mechanical installations appear in nearly every module. Furthermore, Table 1 clearly shows the cohesion between the spatial classification chosen to determine the modules and the NL$\mathrm{SfB}$ construction classification. When a customer wants to change a certain module, Table 1 shows which construction elements might change with it.

\subsubsection{Determine the lay-out}

The layout of the product, in this case a house, shows how the components are coupled. The layout was already derived from specifying the modules and their functions; see Fig. 2. Martin and Ishii (2002) also recognized the importance of determining the layout of the modules and took this step as the first step in determining the coupling index (CI). 


\subsection{Determine the interfaces}

In this subsection, we present how the DFV design steps were adapted to the specific housing design context of Plegt-Vos Living.

\subsubsection{Generate the generational variety index (GVI)}

The first step in DFV is to estimate the generational variety index (GVI). The GVI indicates which components are likely to change over time. It is an indicator of the amount of redesign required for a component to meet the anticipated market requirements. The GVI is based on an estimate of the required changes in a component resulting from external (i.e., non controllable) factors. Examples of such external drivers include customer needs, reliability requirements and reduced prices. (Martin and Ishii 2002). The GVI is estimated in six steps:

1. Determine market needs

2. Create quality function deployment (QFD) matrix

3. List expected changes in customer requirements

4. Estimate engineering metric target values

5. Create GVI matrix

6. Calculate GVI.

Step 1: Determine market needs Plegt-Vos Living did not have specific information about its customers' needs. We therefore used data from Hofman et al. (2006). In his research, Hofman investigated which components or subsystems of the house the customer wants to have the opportunity to specify. For every aspect, Hofman investigated its importance to the customer and how customers prefer to be involved in the design and execution phase of their homes. The Hofman study was based on a vignette-based survey (e.g. Rossi 1982; Govers 1993; Wason et al. 2002) in The Netherlands of which the sampling frame consisted of 304 potential buyers of new homes. On a vignette, personal or social situations are represented by some short descriptions. The descriptions reflect the most important factors in the priority decision-making process and each description contains a well-defined stimulus component. Vignette-based studies are superior to direct-question-based studies because vignettes better approximate real-life decision making situations (Wason et al. 2002).

Step 2: Create the QFD matrix Quality function deployment (Akao and Mazur 1990) is used by many design teams to translate customer requirements into inputs for product designs and organizational processes. For the QFD matrix in this research, the aspects that customers want to have the opportunity to alter, are taken from Hofman et al. (2006), and are expressed as customer requirements. As shown in Table 2, these requirements are directly coupled to the modules. For instance, input to the aspect 'kitchen' belongs to the module kitchen. In addition, the wish to choose type, location and number of electric sockets and switches applies to all modules which contain these components. However, the module's dormer, bay window, garage and storage were not included in Hofman's research. These options are coupled here to the aspect accessory structure.

Step 3: List the expected changes in customer requirements The third step is to estimate qualitatively (high/medium/low) the range of change for the customer requirements. Customers value the opportunity to alter several aspects of the building. The weight given to every aspect is then used in this step. The importance is rated in percentages, with $0 \%$ meaning neutral interest and $100 \%$ meaning very important.

Step 4: Estimate the preference type of involvement Hofman et al. (2006) distinguishes three ways of participation: free choice, choice among alternatives and choice by an expert. The column to the right in Table 2 shows for every aspect in which way customers prefer to participate. The length of the bar indicates the percentage of respondents preferring each particular way of involvement. For instance, it shows that most respondents prefer free choice in the type of kitchen, some prefer choice from alternatives and that only a few are happy to leave the decision to an expert. Where aspects are considered to be of less importance, customers in majority are content to select from alternatives.

Step 5: Create GVI matrix For every coupling between aspect and module (step 2), the weight of importance of customer involvement (step 3 ) is added, as can be seen in Table 2. In this research, the relations between the involvement aspects and the modules all have the same value. This is based on the assumption that the weight for involvement for an aspect is the same for every module. For instance, a customer's preference to choose the sanitary ware (types and colour of toilet, washbasin, etc.) is assumed to be of equal importance to the bathroom as to the toilet in the hallway.

Step 6: Calculate the GVI The final step is to calculate the GVI. The GVI for each component is calculated by summing each of the columns of the GVI matrix. The total for each module indicates the importance of involvement in the module. The GVI in the research conducted by Martin and Ishii indicates which components need to be adjusted to answer future demands. The GVI in this research indicates which module needs to be adjusted with the involvement of the 
Table 2 GVI calculation for Plegt-Vos Living design concept
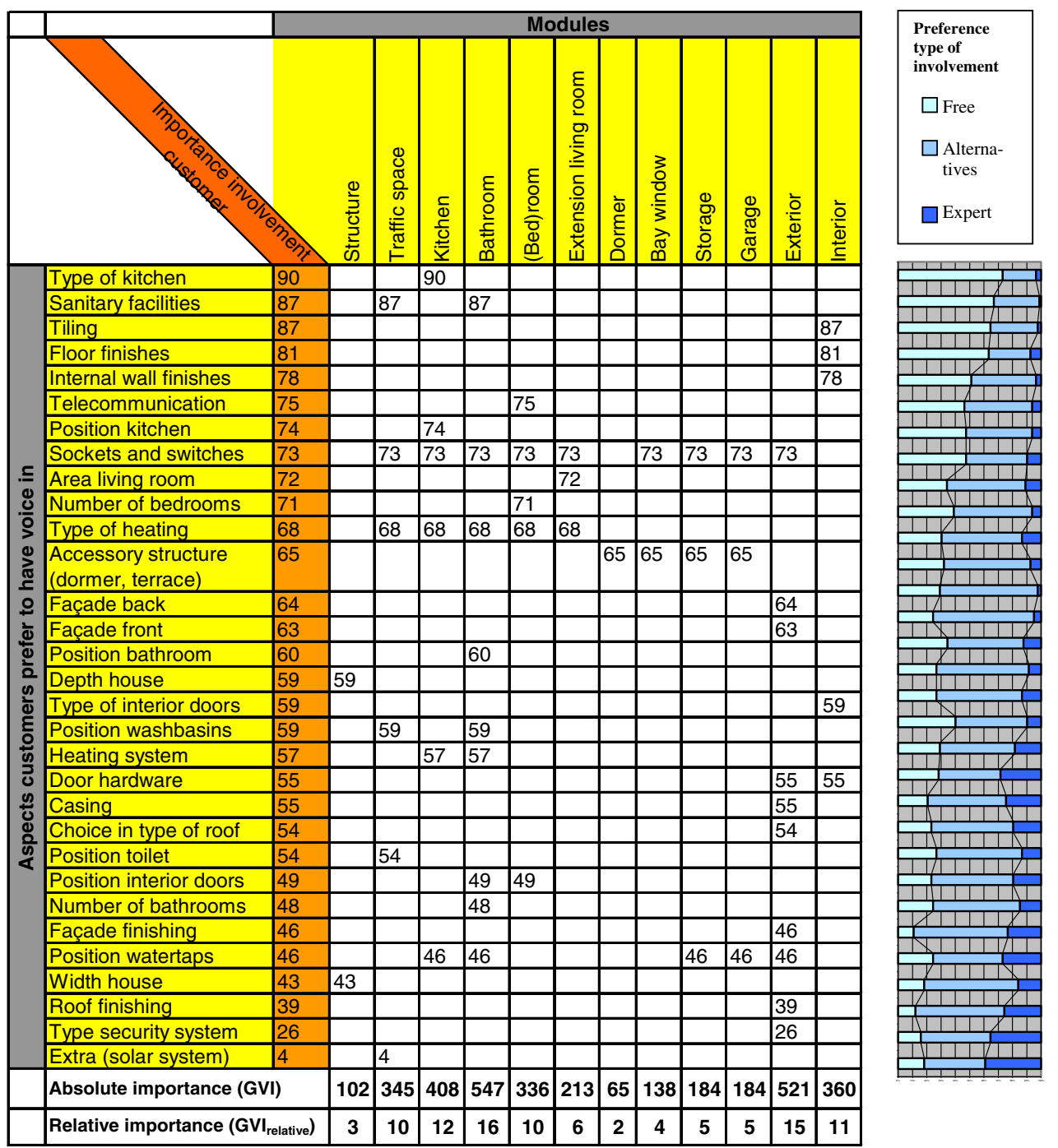

customer. For each result, the relative importance is given. This is calculated as follows:

$\mathrm{GVI}_{\text {relative }}=\frac{\mathrm{GVI}_{\mathrm{i}}}{\Sigma \mathrm{GVI}_{\mathrm{i}}} * 100$.

Dividing the absolute importance for a module by the total sum of absolute importance of all modules $(3,403)$ times 100 gives the result for $\mathrm{GVI}_{\text {relative }}$ The relative importance shows a clearer view on the importance among modules. Table 2 shows that customers tend to prefer the highest involvement in the selection of bathrooms, exteriors and kitchens.

\subsubsection{Estimate the coupling index (CI)}

The coupling index (CI) is estimated in the following three successive steps:
1. List the specification flows between components 2. Estimate the sensitivity of components to changes 3. Calculate the coupling index.

Step 1: List the specification flows between components This step focuses on the relation between components. According to Martin and Ishii (2002) specification flows are defined as the design information that must be passed between designers to help them design their respective components. By mapping out the specification flows early in the design process, the team explicitly describes the relationships that couple the parts. For each control volume, the design team has to list the specifications they need to receive from each of the other control volumes, the coupling index-receiving (CI-R) and the specifications that they expect to supply to each of the other control volumes, and the coupling index-supplying (CI-S). An 
overview is shown in Table 3. The top row of the matrix lists the components supplying the information; the left column lists the components receiving (or requiring) the information. For instance, a change in the hallway, defined as the traffic module, from the front entrance to the side entrance changes the position of the external wall opening for the entrance door in the structure module.

Step 2: Estimate sensitivity of components to changes The sensitivity of components to changes is estimated by evaluating the specification flows. For each specification, the team estimates the sensitivity of each component to a small change in that specification. If a small change in the specification requires a change in the component, then the component has a high sensitivity. If the specification requires a large change to create a change in the receiving component, then it has a low sensitivity. For example, take the specification flows between the structure and bathroom modules; see Fig. 3. A change in the bathroom module alters the position and dimensions of the window and the position of pipe works and wiring. Repositioning of pipes in the main structure is often difficult. Applying the systems wall eases this. The total rating for each specification flow is given in Table 3.

Step 3: Calculate coupling index In step 1, it became clear that the coupling index consists of two indices: the CI-R and the CI-S. The CI-S and CI-R indicate how tightly coupled a component is. With the help of the matrix shown in Table 3, the indices can be easily calculated. The sum for a row is information being received by each component, the CI-R. The sum for a column indicates the strength (or impact) of the information supplied by that component to other components, the CI-S. For instance, the CI-R for the exterior is high, which means that the other components have a strong impact on it. The structure has the highest CI-S, indicating that its design has the strongest impact on other components. The full coupling index results are shown in Table 3.

\subsubsection{Order the modules}

The GVI and the CI taken together, give a better understanding of the influence of external factors and the way in which changes affect the product design. This next step will focus now on the attribution of indices to the set up of a platform that is more resistant to external changes. To this end, components are ranked from highest to lowest, based on the GVI, as shown in Table 4. These are the modules that customers prefer to have most involvement in specifying and therefore are most likely to change.
Martin and Ishii (2002) suggest plotting the GVI-CI indices as a visual aid. Figure 4 shows CI-R values plotted against the corresponding GVI. GVI is an indicator of the external drivers of change (customer requirements), while CI-R is a measure of the internal drivers. So this graph is an overall indicator of how much a component is expected to change. The purpose of the two lines is explained in Sect. 4.3. The "bubble" graph in Fig. 5 adds CI-S to the graph, represented as the size of the bubble. The GVI and CI-R values give an indication of how much the component is expected to change, and the CI-S is a measure of how likely those changes also affect other components.

\subsubsection{Determine where to focus efforts}

The goal of the design team is to design the product platform in such a way that, as much of the design as possible is standardized across generations. For the parts of the design that cannot be standardized, the team should aim to modularize them. To determine which components can be standardized or modularized we followed the decision rules suggested by Martin and Ishii (2002) and described below.

Standardized parts (GVI and CI-R related):

- Fully standardized: it is expected that the component will not change across generations. This implies that the GVI and CI-R are both equal to zero.

- Partially standardized: the component is expected to require minor changes across generations. The higher the GVI and CI-R, the less standardized is the component.

Modularized parts (CI-S related):

- Fully modularized: the geometry, energy, material, or signal (GEMS) of the component can be changed to meet expected customer requirements without requiring other components to be changed. This implies that the CI-S of the component is zero.

- Partially modularized: changes in the GEMS of the component may require changes in other components. The higher the CI-S, the more the changes expected, and thus the component is considered to be less modular.

In terms of procedure, Martin and Ishii suggest that design teams should first consider the components with high GVI values, since these components require high redesign efforts due to changing customer demands. While high CI-R components can also require high redesign efforts, the high GVI components generally will have a much greater impact on the redesign ef- 


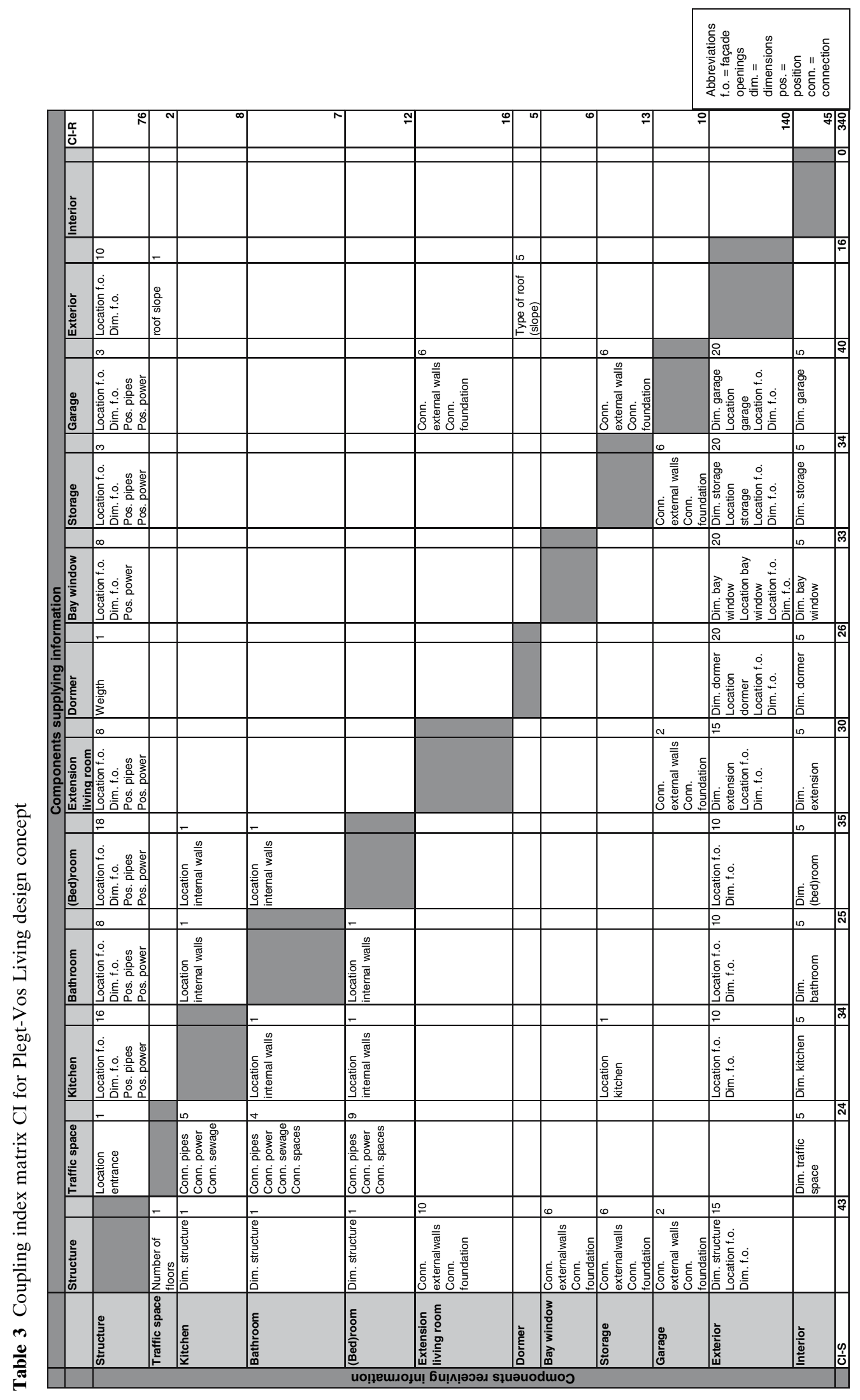




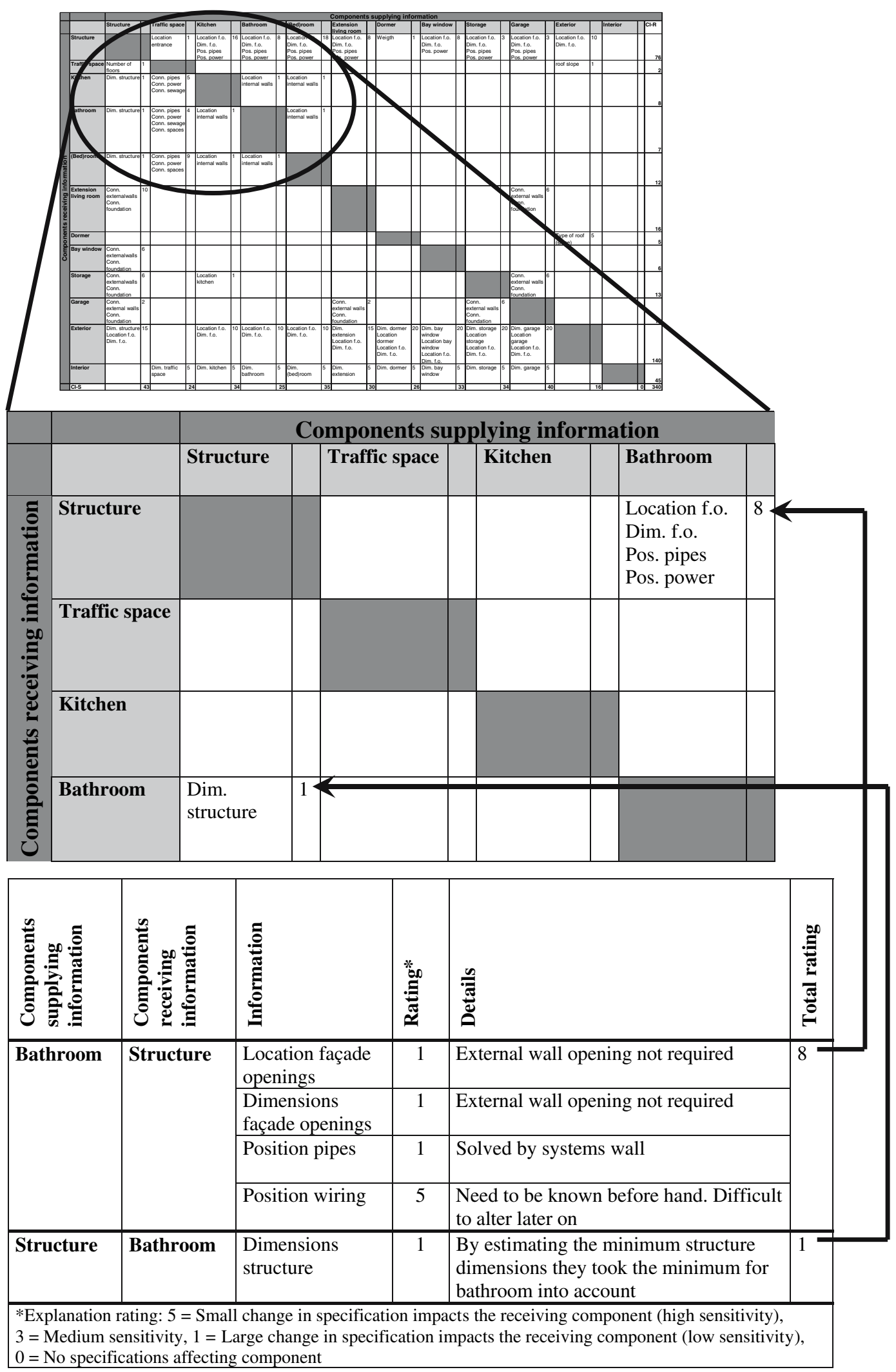

Fig. 3 Estimating sensitivity of specifications between structure and bathroom 
Table 4 Modules sorted by GVI

\begin{tabular}{llll}
\hline Module & GVI & CI-R & CI-S \\
\hline Bathroom & 547 & 7 & 25 \\
Exterior & 521 & 140 & 16 \\
Kitchen & 408 & 8 & 34 \\
Interior & 360 & 45 & 0 \\
Traffic space & 345 & 2 & 24 \\
Bed(room) & 336 & 12 & 35 \\
Extension living room & 213 & 16 & 30 \\
Storage & 184 & 13 & 34 \\
Garage & 184 & 10 & 40 \\
Bay window & 138 & 6 & 33 \\
Structure & 102 & 76 & 43 \\
Dormer & 65 & 5 & 26 \\
\hline
\end{tabular}

forts. Another consideration is to standardize high CI$\mathrm{S}$ components since these have a high potential for causing changes in other components. However, it is not always possible to standardize components. Complete standardization of a product is never possible since it is inevitable that some of the components must change in order to meet changing customer requirements.

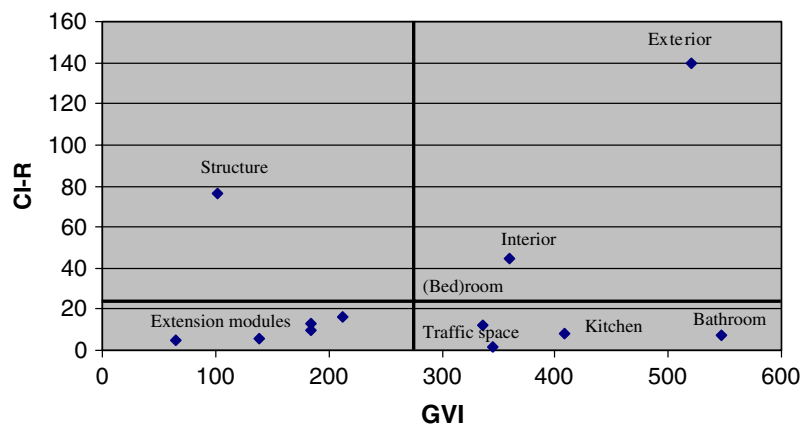

Fig. 4 Coupling index-receiving versus generational variety index

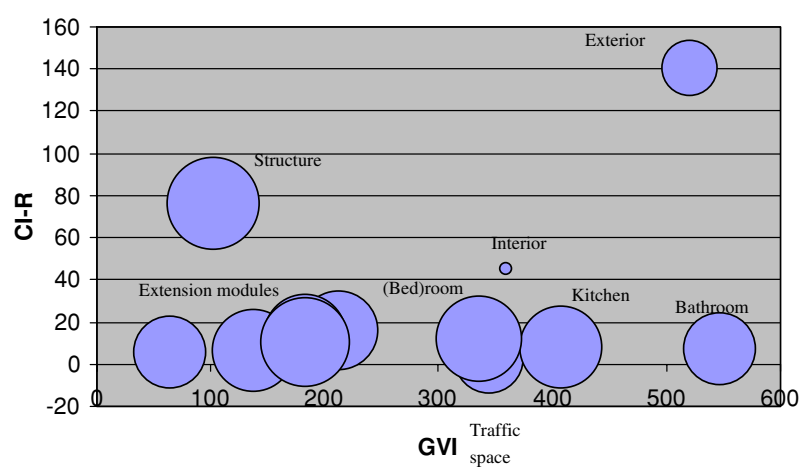

Fig. 5 Coupling index-supplying versus coupling index-receiving versus generational variety index

\subsection{Determine standards}

The GVI, CI-R and CI-S rankings are used to determine which components can be standardized or modularized to achieve an effective platform. To realize this, the components are categorized into high/low categories. The design team is free to define the demarcation line between high/low. The team could choose the median, the top quartile, and so on as the demarcation. In this research, demarcations for both the GVI and the CI-R values are based on the average of each indicator. In the Plegt-Vos Living case, the average GVI is 284 and CI-R is 28 . These demarcation lines are show in Fig. 4.

Since this research focuses on meeting customer demands for involvement in several aspects of the house, it is proposed that modules with high GVI should achieve a high level of modularization and that the low GVI modules should be standardized.

As Fig. 4 shows, both the GVI and the CI-R values of the extension modules are low. The low GVI value indicates that customers prefer hardly any influence on the design of these modules. The low CI-R value indicates a low sensitivity to changes to other modules. The low CI-R value can be explained by Plegt-Vos Living's standardization of most aspects of these modules. Low values for GVI and CI-R indicate that these modules can be standardized.

The structure module has a low GVI value as well; however the CI-R is relatively high. This means that customers prefer little involvement, but standardization is more difficult mainly due to the built-in modules, as can be seen in Table 3. However, standardizing this module is strongly recommended, since it has high CI-S and therefore a high potential for causing changes in other components. Conversely, the built-in modules and the traffic module have a high GVI and a low CI-R. These modules are straightforward to standardize, but the customer prefers most involvement in these modules, so it is recommended that these should be modularized. Finally, the interior and exterior modules finally have both a high GVI and high CI-R value, but a relatively low CI-S value. The customer prefers involvement in these modules, and these modules are straightforward to modularize (i.e., lower the CI-S). The high CI-R can be a sign to try to overlook the internal couplings once more in order to lower the CI-R.

With regards to product family design, the GVI, CI$\mathrm{R}$ and CI-S rankings can be used to balance the commonality potential and differentiation needs within a product family. By categorizing the components into high/low categories, the design team separates the common (platform) elements, i.e. the components that 
are suggested to be standardized, namely the components with the low GVI, from the differentiating (nonplatform) elements, i.e. the components customers prefer most involvement in, i.e. the components with the high GVI. From a design point of view, the CI-R and CI-S rankings indicate the difficulty of standardizing or modularizing the components.

Plegt-Vos Living has already taken a few steps towards standardization. By defining standard measures and standardizing connections such as floor-wall connections, the two structural materials (concrete and sand lime-stone), can be easily substituted for each other. Furthermore, Plegt-Vos Living has classified standard steps to broaden and lengthen the house. The length and width of the floor slabs are taken into account. These slabs are $1.2 \mathrm{~m}$ wide and can be cut by the supplier to any desired length of floor up to $7.2 \mathrm{~m}$. The floor slabs are placed crosswise and the minimum length of each house is $10.2 \mathrm{~m}$, which is eight and a half slab lengths. The minimum width is $5.4 \mathrm{~m}$ and can be widened in $0.3 \mathrm{~m}$ steps. The measure of $0.3 \mathrm{~m}$ is chosen by Plegt-Vos Living as a modular size, since this is widely used in the construction industry, for instance for placing roof tiles. The house can be lengthened in steps of $0.6 \mathrm{~m}$. corresponding to half the width of a floor slab and is a multiple of $0.3 \mathrm{~m}$.

Suggested further steps to standardize the structure module would be to lower the CI-R caused by changes in location and dimension of façade openings and by the position of pipes and cables. The information about these aspects is mainly derived from the in-built modules. So, finding a solution for decreasing the impact of these specifications can also contribute to increasing the level of modularisation of the in-built modules, since it lowers their CI-S values.

\section{Contributions, limitations and future research}

This study contributes to both product development literature and housing literature. The results also hold important guidelines for developing product platforms in the specific setting of the housebuilding industry. Based on the limitations and implications inherent in this study, future directions for research are also proposed.

\subsection{Contributions to the product development} literature

Complex products and systems (CoPS) have been identified as a distinct area of innovation research. For CoPS, the variety of subsystem combinations can cause high levels of uncertainty and risk in system design, production and integration (Hobday 1998; Hobday et al. 2000). Reduction of this uncertainty and risk can be realized by moving beyond the creation of projectspecific unique solutions. Key in this approach is the sharing of components, modules and other assets across multiple projects. Not withstanding the body of literature concerning the concepts of modularity and product platforms and their application in practice, there is a gap in the literature when it comes to their applicability to CoPS. Production of the built environment, as a subset of CoPS, involves making many types of technically and organizationally complex products such as hospitals, concert halls, research laboratories and silicon fabrication plants (Gann and Salter 2000). In this study, we have contributed to filling this gap in the literature by investigating the potential of module-based platform concepts for the housebuilding industry. Implementing the platform concept significantly can significantly increase cost and time efficiencies, technological leverage and market power. By using standardized and pretested components and modules, substantial reductions in decision making and uncertainty can be realized. The accumulated learning and experience may in general, also result in higher product performance, causing fewer mistakes and quality failures, and lower rework costs. Josephson and Hammarlund (1999) and Love (2002) report that the rework costs of residential, industrial and commercial building projects range from 2 to $6 \%$ of their contract values. Yet there is a great need to reduce rework costs if projects are to improve their productivity and performance.

So far, no systematic engineering design methods have been applied specifically in the housebuilding industry setting that link product platform development with customer requirements. The available literature on platform-based strategies has, for the most part, focused on the concepts and benefits of platform development. This research has contributed a methodology to the literature for developing product platform architectures in the specific setting of the housebuilding industry. By providing general variety and coupling indices to compare product architectures along dimensions of interest, the applied method can be characterized in the terms described by Fixson (2005) as an engineering design method. This engineering based methodology brings together market and product considerations on the decision as to which products to develop. Moreover, the model shows that products can exhibit different degrees of being coupled by determining which components can be standardized or modularized: a product can be characterized by 
different interface characteristics. The different interface characteristics can be related to the problem of 'interface reversibility' as discussed by Fixson (2005). This concept is based on the notion that various product changes over the product life, such as upgrades, add-ons, adaptation, wear, consumption or reuse strongly depend on the so-called reversibility of the interface (ibid., p. 357). The method contributes to an approach that ties the individual components of a building to the different life-cycles they belong to.

\subsection{Contributions to the housing literature}

'Open Building' based on ideas of Habraken (1972) can be seen as an attempt to integrate industrial housebuilding and user participation in house design. The levels of the city structure, urban tissue, support, and infill are usually distinguished. Open Building reflects the idea that the need for change at a lower level emerges faster then at upper levels. Open Building aims at a situation where decisions made at upper levels leave the contents of the decisions made at lower levels open. In order to provide subsequent occupants with the opportunity to modify parts of their interior house design, the elements decided by the resident must be easy to change. In the concept of Open Building, a building structure design is therefore divided into two parts: the chassis (also called support) and the infill. The chassis is the standardized, mass produced part of the system and provides both the structure and services. The infill that attaches to the chassis is the customized part and makes up the walls and floors and parts of the building the homeowner sees and interacts with. This is similar to the specification of the structure module and the inbuilt modules in the Plegt-Vos Living study. Our methodology offers those building companies that apply the Open Building concept a tool to further refine the modularization of the infill components and their interfaces to the chassis.

This study also provides a distinct way of housing classification. As explained in the theoretical section, the traditional way of classifying a building is to decompose the building according to its technical construction elements. To improve communication between the housebuilding industry and its customers, we have developed a classification system according to the spatial use in houses: multifunctional rooms, rooms with a specific use, secluded rooms and supplementary rooms. The methodology subsequently suggests how to determine the construction elements of each module as well as the relationship between modules, leading to specifications concerning lay out needs.

\subsection{Managerial implications}

Taken as a whole, this article provides practical guidelines and decision rules to help housebuilding companies develop their product platform architectures effectively. Based on the GVI, CI-R and CI-S index values generated for each module, a design team can determine which modules to standardize or further modularize to achieve an effective platform. Besides the step-to-step guidelines, plotting the indices from the DFV method was found to be a very useful visual aid in the Plegt-Vos Living study.

Our method can further help a company to understand the commonalities that tie the firm's offerings, and exploit these to create variety that customers value. Furthermore, the proposed approach to take into account the customers' perspective and requirements concerning customizing certain aspects of the housing design, provides building companies with a useful tool in improving communication with customers and translating their needs into solutions that match the individual preferences and tastes. The spatial module classification has demonstrated its usefulness in this respect. This approach is new to most building companies that have been used to mass-producing standard houses with little direct customer input for many years.

\subsection{Limitations and future directions for research}

In this research, an in-depth study application was carried out in only one housebuilding company. The results of this study have been presented to a network of housebuilding companies. The network members recognized the applicability of the proposed methodology and have started pilot projects to investigate its added value for their companies. Future research will report on the findings of these pilot projects. Although only the housebuilding industry has been the focus in this research, one might also consider extending the research to test the applicability of the methodology in comparable building industries such as the development and construction of offices and other utility buildings. By understanding and focusing on the different organizational contexts in which building platforms are applied, future research may develop categories of options for platform development that are useful in practice, given a specific context.

This study has focused on product platform design. However, heterogeneous markets are increasingly forcing many companies to simultaneously compete in the three domains of product, process and supply chain (Fixson 2005). By allocating products to the appropriate process platform, manufacturers can effectively 
manage the contradictions between high-volume and high-flexibility manufacturing (Sawhney 1998). The interface characteristics of a product can affect a firm's sourcing strategy: outsourcing commodity items while keeping components of strategic importance in-house (Fixson 2005). According to Fixson, the type of interfaces between components can also affect the extent to which strategies for postponement and late customization can be realized. Further research is suggested therefore to also investigate options to apply the platform concept in the manufacturing and supply chain management process of the building industry.

We have reported the potential benefits of cost reductions caused by an expected decrease in mistakes, quality failures and rework. However, the complex relationship between product architecture and costs is still insufficiently understood (Simpson et al. 2006). Therefore, it is proposed to extend this study by developing a better understanding of this relationship.

The methodology described in this article, has the potential to assist companies in clearly defining a product platform, which accounts not only for efficiency improvement, but also for a better communication with customers. Filling the aforementioned gaps in literature would be an important future contribution, both from the academic and the managerial points of view. We are confident that our research has laid a useful foundation for expanding the investigation to other building processes so as to broadening knowledge about platformbased product architectures in construction.

\section{References}

Akao Y, Mazur GH (1990) Quality function deployment: integrating customer requirements into product design. Productivity Press, Cambridge

Baldwin CY, Clark KB (2000) Design rules: the power of modularity. MIT, Cambridge

Ball M (1999) Chasing a snail: innovation and housebuilding firms' strategies. Hous Stud 14(1):9-22

Barlow J, Childerhouse P, Gann D, Hong-Minh S, Naim M, Ozaki R (2003) Choice and delivery in housebuilding, lessons from Japan for UK housebuilders. Build Res inf 31(2):134-145

Craig D, Roy R (2004) Developing a customer-focused culture in the speculative housebuilding industry. Total Qual Manage Bus Excel 15(1):73-87

DTI (Department of Trade and Industry) (2004) Modern methods of construction in Germany, playing the off-site rule. Report of a DTI Global Watch Mission, London, 76

Erixson G (1998) Modular function development (MFD), Doctoral thesis KTH Royal Institute of Technology, Department of Manufacturing Systems, Stockholm

Fixson SK (2002) The multiple faces of modularity-an analysis of a product concept for assembled hardware products, working paper. MIT, Cambridge
Fixson SK (2005) Product architecture assessment: a tool to link product, process and supply chain design decisions. J Oper Manage 23(3):345-370

Gann DM (1996) Construction as a manufacturing process? Similarities and differences between industrialized housing and car production in Japan. Constr Manag Econom 14(5):437-450

Gann DM, Salter AJ (2000) Innovation in project-based, serviceenhanced firms: the construction of complex products and systems. Res Policy 29(7):955-972

Gibb A (2001) Standardization and pre-assembly-distinguishing myth from reality using case study research. Constr Manage Econom 19(3):321-330

Govers CPM (1993) Quality of services "applicable to production?”. Int J Prod Econ 30-31:385-397

Habraken NJ (1972) Supports-an alternative to mass housing. The Architectural Press, London

Halman JIM, Hofer AP, Vuuren W. Van (2003) Platform driven development of product families, linking theory with practice. J Prod Innovat Manage 20(2):149-162

Hobday M (1998) Product complexity, innovation and industrial organization. Res Policy 26(6):689-710

Hobday M, Rush H, Tidd J. (2000) Innovation in complex products and systems. Res Policy 29(7/8):793-804

Hofer AP, Halman JIM (2005) The potential of layout platforms for modular complex products and systems, J Eng Des 16(2):237-258

Hofman E, Halman JIM, Ion RA. (2006) Variation in housing design: identifying customer preferences. Hous Stud 21(6):931-945

Jones N (2003) Competing after radical technological change: the significance of product line management strategy. Strateg manage J 24(13):1265-1287

Josephson PE, Hammarlund Y (1999) The causes and costs of defects in construction. A study of seven building projects. Automation Constr 8(6):681-642

Kendall S (1999) Open building: an approach to sustainable architecture. J Urban Technol 6(3):1-16

Krishnan V, Gupta S (2001) Appropriateness and impact of platform-based product development. Manag Sci 47:22-63

Love PED (2002) Influence of project type and procurement method on rework costs in building construction projects. J Constr Eng Manage 128(1):18-29

Martin MV, Ishii K (2002) Design for variety: developing standardized and modularised product platform architectures. Res Eng Des 13:213-235

Meyer MH, Utterback JM (1992) The product family and the dynamics of core capability. Sloan Manage Rev 34(3):29-47

Meyer MH, Lehnerd AP (1997) The power of product platforms: building value and cost leadership. Free Press, NY

Meyer MH, DeToreb A (2001) Creating a platform-based approach for developing new services. J Prod Innov Manage 18(3):188-204

Meyer MH, Tertzakian P, Utterback JM (1997) Metrics for measuring research and development in the context of the product family. Manage Sci 43(1):88-111

Muffato M, Roveda M (2000) Developing product platforms: analysis of the development process. Technovation 20(11):617-630

Noguchi M (2003) The effect of the quality-oriented production approach on the delivery of prefabricated homes in Japan. $\mathbf{J}$ Hous Built Environ 18(4):353-364

Ozaki R (2003) Customer-focused approaches to innovation in house building. Constr Manage Econom 21(9):557-564

Rossi PH, Anderson AB (1982) The factorial survey approach: an introduction, In: Rossi PH, Nock SL (eds) Measuring 
social judgments: the factorial survey approach. Sage Publications, Beverly Hills

Roy R, Brown J, Gaze C (2003) Re-engineering the construction process in the speculative house-building sector. Constr Manage Econom 21(2):137-146

Salvador F, Forza C, Rungtusanatham M (2002) Modularity, product variety, production volume, and component sourcing: theorizing beyond generic prescriptions. J Oper Manage 20(5): 549-575

Sanchez R, Mahoney JT (1996) Modularity, Flexibility, and Knowledge Management in product and organization design. Strateg Manage J 17(Winter Special Issue):63-76

Sawhney MS (1998) Leveraged high-variety strategies: from portfolio thinking to platform thinking. J Acad Manage Sci 26(1):54-61

Schilling MA (2000) Towards a general modular systems theory and its application to interfirm product modularity. Acad Manage Rev 25(2):312-334
Simpson TW, Siddique Z, Jiao J (2006) Product platform and product family design, methods and applications. Springer Science and Business Media, New York

Thillart CCAM Van den (2004) Customised industrialisation in the residential sector. SUN Publishers, Amsterdam

Ulrich K (1995) The role of product architecture in the manufacturing firm. Res Policy 24(3):419-440

Voordijk H, Meijboom B, De Haan J (2006) Modularity in supply chains: a multiple case study in the construction industry. Int J Oper Product Manage 26(6):600-618

Wason KD, Polonsky MJ, Hyman MR (2002) Designing vignette studies in marketing. Australas Mark J 10(3):41-58

Wolters MJJ (2001) The business of modularity and the modularity of business, $\mathrm{PhD}$ thesis, Erasmus University Press, Rotterdam 